\title{
Temporal Evolution of the Solar Wind Bulk Velocity at Solar Minimum by Correlating the STEREO A and B PLASTIC Measurements
}

\author{
A. Opitz • R. Karrer • P. Wurz • A.B. Galvin • P. Bochsler • L.M. Blush • H. Daoudi • \\ L. Ellis · C.J. Farrugia - C. Giammanco - L.M. Kistler · B. Klecker · H. Kucharek • \\ M.A. Lee · E. Möbius · M. Popecki • M. Sigrist · K. Simunac $\cdot$ K. Singer • \\ B. Thompson · R.F. Wimmer-Schweingruber
}

Received: 6 November 2008 / Accepted: 14 December 2008 / Published online: 28 January 2009

(C) Springer Science+Business Media B.V. 2009

\begin{abstract}
The two STEREO spacecraft with nearly identical instrumentation were launched near solar activity minimum and they separate by about $45^{\circ}$ per year, providing a unique tool to study the temporal evolution of the solar wind. We analyze the solar wind bulk velocity measured by the two PLASTIC plasma instruments onboard the two STEREO spacecraft. During the first half year of our measurements (March-August 2007) we find the typical alternating slow and fast solar wind stream pattern expected at solar minimum. To evaluate the temporal evolution of the solar wind bulk velocity we exclude the spatial variations and calculate the correlation between the solar wind bulk velocity measured by the two spacecraft. We account for the different spacecraft positions in radial distance and longitude by calculating the corresponding time lag. After adjusting for this time lag we compare the solar wind bulk velocity measurements at the two spacecraft and calculate the correlation between the two time-shifted datasets. We show how this correlation decreases as the time difference
\end{abstract}

STEREO Science Results at Solar Minimum

Guest Editors: Eric R. Christian, Michael L. Kaiser, Therese A. Kucera, O. C. St. Cyr

A. Opitz · R. Karrer · P. Wurz · P. Bochsler · L.M. Blush · H. Daoudi · C. Giammanco · M. Sigrist Department of Space Science and Planetology, Physics Institute, University of Bern, Bern, Switzerland

A. Opitz $(\bowtie)$

Centre d'Etude Spatiale des Rayonnements (CNRS-UPS), University of Toulouse, Toulouse, France e-mail: opitz@cesr.fr

A.B. Galvin · L. Ellis · C.J. Farrugia - L.M. Kistler · H. Kucharek · M.A. Lee · E. Möbius · M. Popecki · K. Simunac $\cdot$ K. Singer SSC, University of New Hampshire, Durham, NH, USA

B. Klecker

MPI for Extraterrestrial Physics, Garching, Germany

B. Thompson

NASA/GSFC, Greenbelt, MD, USA

R.F. Wimmer-Schweingruber

Institute for Experimental and Applied Physics, University of Kiel, Kiel, Germany 
between two corresponding measurements increases. As a result, the characteristic temporal changes in the solar wind bulk velocity can be inferred. The obtained correlation is 0.95 for a time lag of 0.5 days and 0.85 for 2 days.

Keywords Solar wind $\cdot$ STEREO $\cdot$ Plasma $\cdot$ Multipoint spacecraft observations

\section{Introduction}

This paper focuses on the solar wind temporal evolution derived from two-spacecraft measurements. Spatial effects are excluded by adjusting for the theoretical time lag calculated from the spacecraft positions and then correlating the two datasets. Previous works applied autocorrelation analyses of observations performed from one vantage point. For instance, Gosling and Bame (1972) studied combined Vela 2 and 3 data by using autocorrelation analysis methods. They found that the solar wind can be considered persistent up to three days and the average correlation between bulk velocity measurements separated in time by one Carrington rotation is 0.3 , by one day it is 0.64 and by three hours it is 0.94 . In a later paper Gosling et al. (1976) analyzed the solar wind velocity variations for a longer time period (1962 - 1974), which involves a whole solar cycle, and found the highest correlations at the declining phase of the solar cycle, where the coefficient exceeds 0.5 for one Carrington rotation. Coplan et al. (2001) studied the correlation of solar wind parameters between SOHO and Wind. They found higher correlations during periods of lower solar activity. In contrast, King and Papitashvili (2005) found by autocorrelation analysis of Wind and ACE data that the plasma structure scales have little or no dependence on solar cycle phase.

Richardson, Dasevisky, and Paularena (1998) have correlated ISEE 3 and IMP 8 plasma data and found that the correlation coefficient decreases as the correlation time period is decreased. Paularena et al. (1998) have correlated IMP 8, Wind, and INTERBALL-1 plasma parameters near solar minimum and analyzed the standard deviation dependence of the correlation coefficient. Coplan et al. (2001) point out that the lower correlation coefficients for higher time resolution data are due to the larger random component and fewer plasma parameter changes. We note here that the latter is simply a mathematical effect and suggests that a dataset with high standard deviation will carry a higher correlation coefficient.

These previous correlation studies used data from spacecraft positioned relatively near each other, with very small longitudinal separation. Since the variation of the measured solar wind velocity at $1 \mathrm{AU}$ stems mainly from a combination of the temporal evolution of the source regions and the solar rotation relative to the observing spacecraft, the rotating solar sources have to be followed to exclude the spatial effect. The temporal evolution of the solar wind bulk velocity can be also inferred if we exclude the effect of the solar rotation by using the measurements of two spacecraft with longitudinal separation. Two spacecraft positioned in the ecliptic with similar radial distance, but with larger longitudinal separation ( $>$ few degrees), carrying identical instrumentation are ideal to do this study. By considering the solar rotation and the two spacecraft positions, solar wind source regions can be followed, and the time scales of the solar wind bulk velocity persistence can be derived.

Such an excellent opportunity to examine the temporal evolution of the solar wind bulk velocity is provided by the two spacecraft of the Solar Terrestrial Relations Observatory (STEREO) mission (Kaiser et al., 2007). STEREO provides a totally new perspective on the Sun and the inner heliosphere by imaging simultaneously from two nearly identical space-borne observatories with an increasing spatial separation. STEREO's primary goal is to advance the understanding of the three-dimensional structure of the solar corona and its 
Figure 1 Sketch of the two STEREO spacecraft positions (A and B) in the ecliptic plane. The time lag from the longitudinal spacecraft separation, $t_{r}$, and the time lag from the radial spacecraft separation, $\Delta t$, are indicated, and $\omega$ refers to the solar rotation.

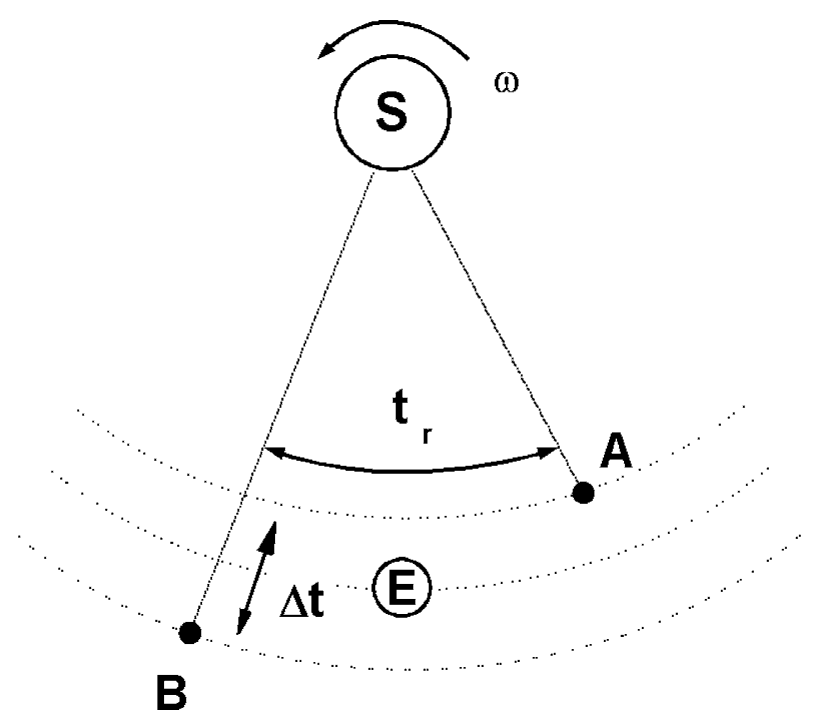

temporal evolution. The two spacecraft were launched together on 25 October 2006 from Cape Canaveral (Florida, USA) onboard a Boeing-built three-stage Delta II rocket. One observatory is placed ahead (A) of the Earth in its orbit and the other behind (B), enabling us to observe the Sun from two vantage points (Figure 1). Both spacecraft are drifting away from the Sun - Earth line, keeping approximately the Sun-Earth distance from our central star (Figure 2, middle panel). The spacecraft separation is growing with time since the A spacecraft is faster (with an orbit slightly closer to the Sun) by $\sim 22^{\circ}$ per year and the B spacecraft is slower (with an orbit slightly farther away from the Sun) by another $\sim 22^{\circ}$ per year (Figure 2, top panel). After launch, the two observatories were close enough for their instruments to be cross-calibrated. Near Earth, however, the two spacecraft often were in different plasma regions (e.g., one in the magnetosheath and the other in the undisturbed solar wind). The first few years of the STEREO observations fall into the solar activity minimum, which enables a two-point correlation analysis from two widely spaced probes of these conditions.

\section{PLASTIC Datasets}

The Plasma and Suprathermal Ion Composition (PLASTIC) experiment (Galvin et al., 2008) is the primary in situ solar wind ion instrument onboard the STEREO spacecraft. PLASTIC's objective is to measure the abundance and charge state distributions of various solar wind species and their velocity distributions with high spatial and temporal resolution. The PLASTIC instrument consists of the Entrance System, which is a hemispherical electrostatic analyzer (ESA), and a time-of-flight mass spectrometer (TOF) with solid state detectors. The Entrance System selects the ions by their energy-per-charge ratio and the out-of-ecliptic angle of their arrival direction (elevation angle or deflection). PLASTIC has three apertures, each with different acceptance characteristics. It is designed to accept solar wind ions through the Solar Wind Sector (SWS) and suprathermal ions using the Wide Angle Partition (WAP). The field of view of the SWS is $\pm 22.5^{\circ} \times \pm 20^{\circ}$ (in- and out-of-ecliptic angles, respectively) and it is always pointing toward the Sun. It has a novel ion-optical design that 

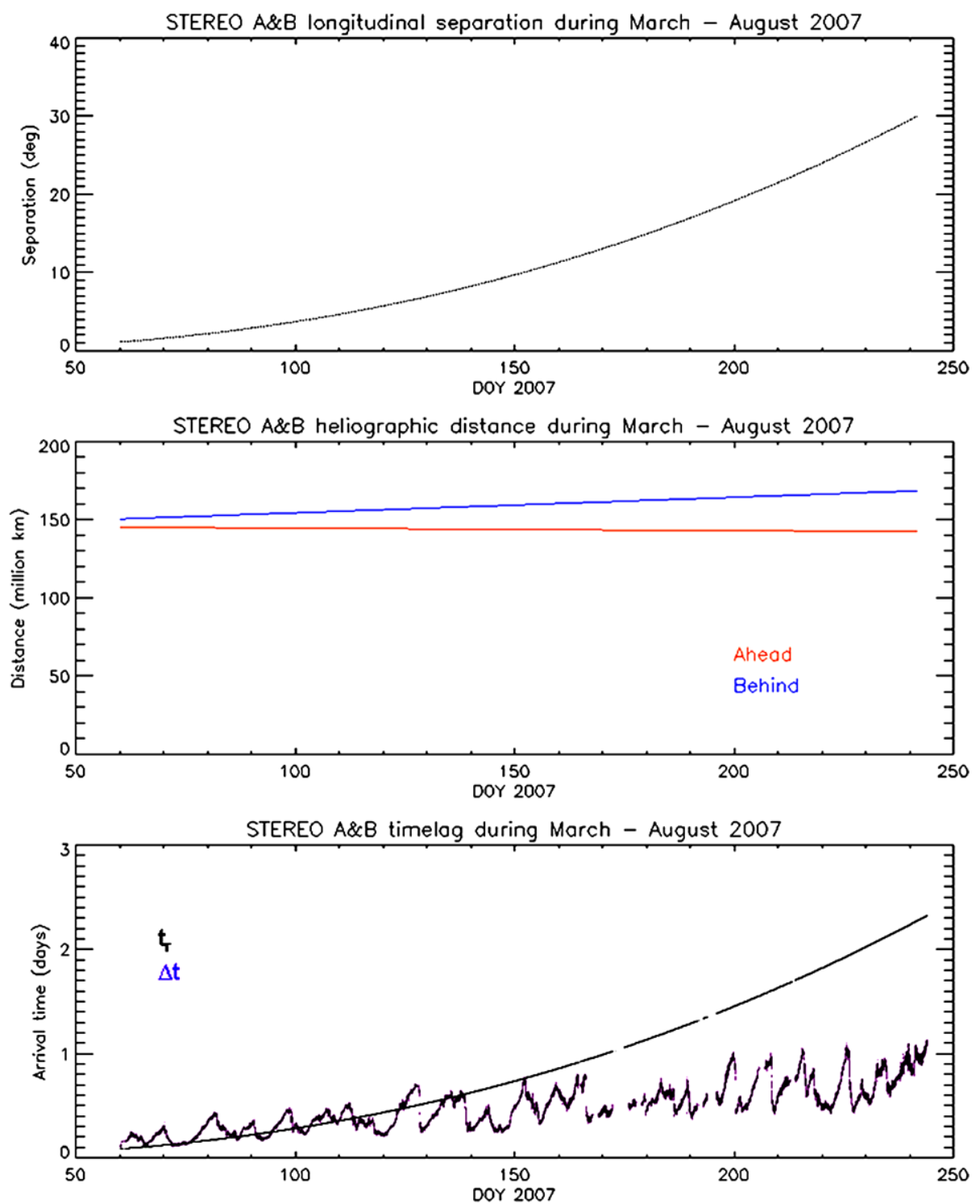

Figure 2 Top: Longitudinal separation of the two STEREO spacecraft during the time period of March-August 2007. Middle: Their radial distance from the Sun for the same time period. Bottom: Theoretical time lag between the two spacecraft from their longitudinal separation, $t_{r}$ (black), and theoretical time lag from their radial separation, $\Delta t$ (violet).

uses two different apertures, one for less abundant and one for more abundant solar wind elements. Lower ion fluxes are measured by the Main channel (MC), whereas higher ion fluxes are measured by the S-shaped channel (SC), which has a much smaller geometric factor. If the count rate measured through the MC exceeds a commandable value, an electronic switch changes to the SC, which reduces the count rate to acceptable values. The SC of the SWS allows the measurement of the distribution functions of solar wind protons and alpha par- 
ticles, providing density, velocity, and kinetic temperature information every minute. Due to its geometry, the ESA focuses the parallel beams into one point at the exit slit. This ion image is preserved throughout the TOF unit and recorded on the detectors. Hence the position on the detector defines unambiguously the in-ecliptic angle of the incident ions. Since STEREO is a three-axis-stabilized, nonspinning spacecraft, the out-of-ecliptic angle information is obtained by applying deflection plates. These are mounted at the entrance of the two SWS apertures and electrically tuned to guide ions from selected elevation angles into the center of the aperture. PLASTIC provides energy spectra and 3D velocity distributions with a one-minute time resolution. From these products solar wind bulk properties can be determined.

The measured raw datasets were converted to physical units by using laboratory (Karrer, 2007) and our in-flight calibration results. For each spacecraft, the solar wind bulk velocity is calculated by fitting a drifting Maxwell - Boltzmann distribution function to the solar wind proton velocity distribution (Opitz, 2007) for every minute. The obtained solar wind bulk velocity is shown on the top panels in Figure 3 for STEREO A (in red) and for STEREO B (in blue). It has a typical solar minimum pattern by varying between slow and fast solar wind velocities $\left(\sim 300\right.$ to $\left.\sim 750 \mathrm{~km} \mathrm{~s}^{-1}\right)$ without any larger transient events. This fact enables the study of a quiet Sun and the nominal solar wind conditions.

We analyzed these PLASTIC solar wind bulk velocity results starting from 1 March (DOY 060) until 31 August 2007 (DOY 243). Although the PLASTIC plasma measurements are available from 20 January 2007, we use only datasets starting later because after launch the B spacecraft remained near Earth for a long period, and hence the solar wind plasma measurements were frequently influenced by the terrestrial magnetosphere. In this paper correlation and regression analyses are performed on these one-minute solar wind bulk velocity datasets measured by the two PLASTIC instruments onboard STEREO.

\section{STEREO Time-Lag Calculation}

We study solar wind bulk velocity temporal evolution at $1 \mathrm{AU}$ by observing plasma corresponding to the same solar origin but emerged at different times. This can be achieved by observing the solar wind bulk velocity from one vantage point and somewhat later from another vantage point following the solar rotation (Figure 1). We are using data from two nearly identical instruments (PLASTIC) located on two different spacecraft (STEREO A and B) with monotonically increasing longitudinal separation $\left(\sim 45^{\circ}\right.$ per year). So the rotational time lag, $t_{r}$, from this increasing longitudinal separation is also increasing, which provides a unique tool to derive the characteristic time scales for the persistence of the solar wind bulk velocity.

In order to derive the temporal evolution we exclude the spatial variations by adjusting for the radial and longitudinal separation of the two spacecraft. We neglect their latitudinal separation because both STEREO spacecraft are nearly in the ecliptic plane. This assumption might introduce an error if the latitudinal velocity gradient is large. Note also that at larger longitudinal separations the two spacecraft might be on different sides of the heliospheric current sheet.

The radial distance of the two STEREO spacecraft from the Sun is approximately 1 AU, but there is a small radial difference between the A and B orbits (middle panel in Figure 2), therefore the solar wind arrival times are slightly different even for the same longitudinal location. When calculating the time lag from this radial separation, $\Delta t$, we assume that the solar wind bulk velocity remains constant over this small distance between the A and B 

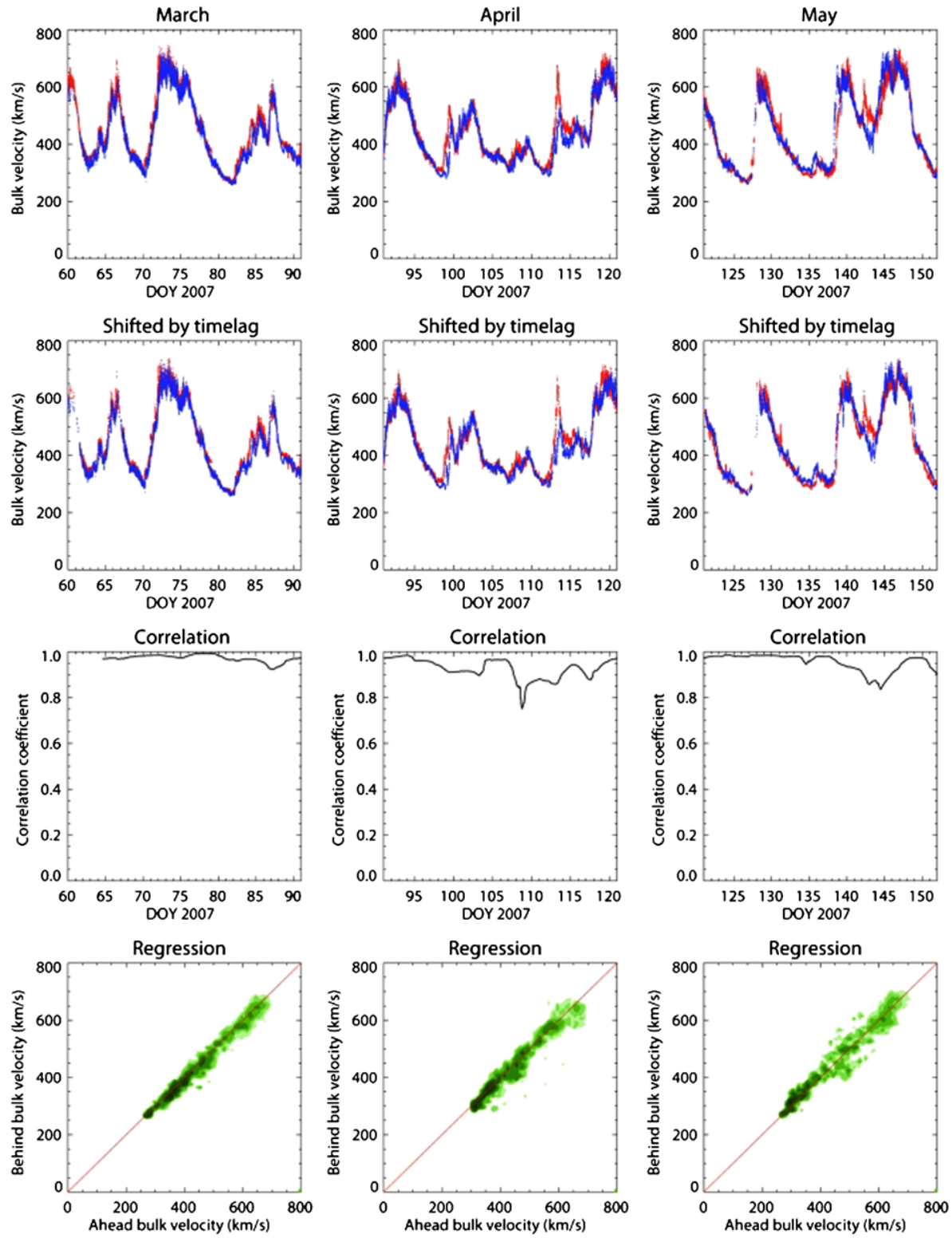

Figure 3a Data plots for March-May 2007. Top row: Calibrated one-minute STEREO A (red) and B (blue) PLASTIC solar wind bulk velocity data. Second row: The same data as in the top row, but shifted by the radial and longitudinal time lag. Third row: Correlation between the A and time-shifted B bulk velocity data over one-third of a Carrington rotation time period (CR/3). Fourth row: Regression plot of the A and time-shifted B bulk velocity data. 

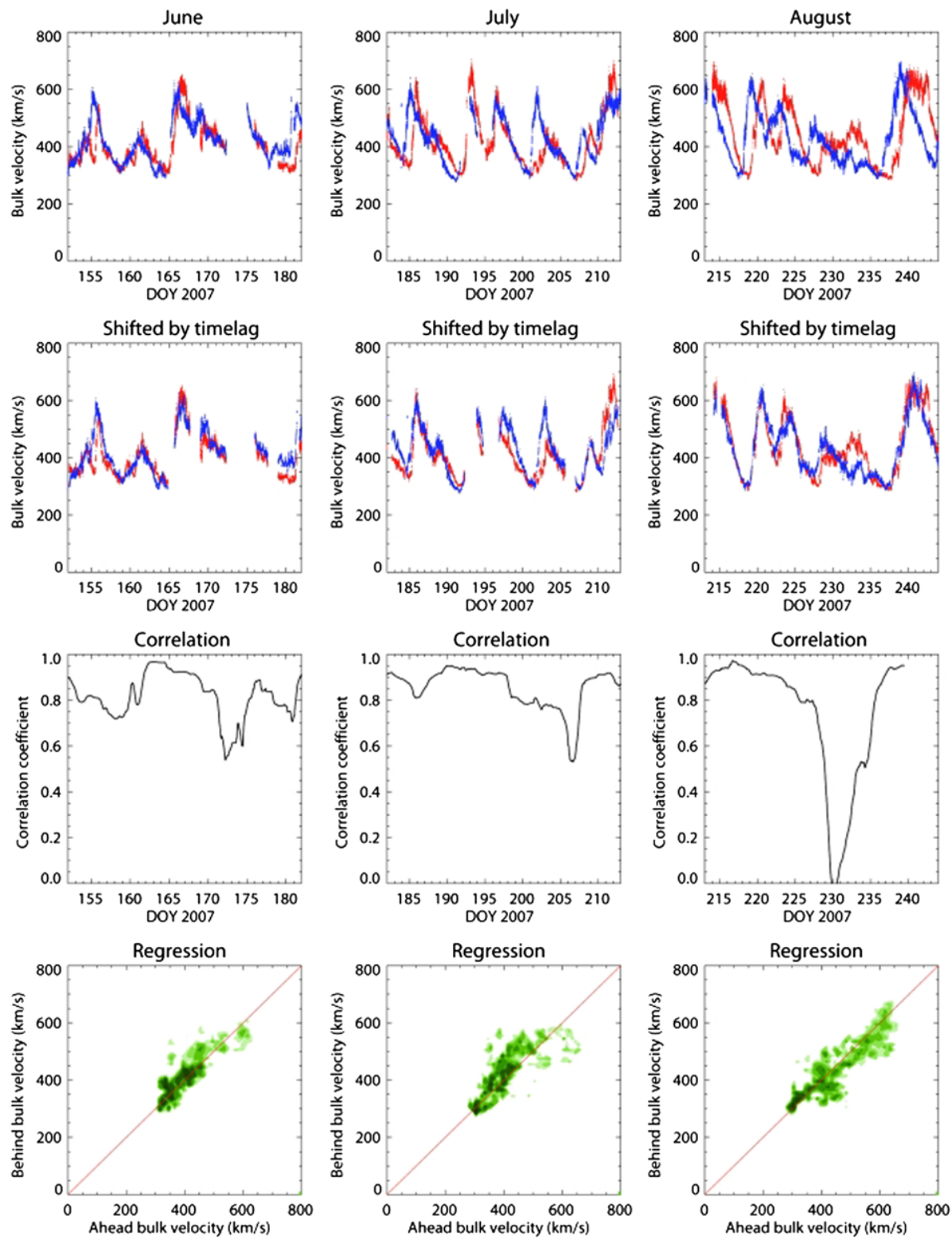

Figure 3b Data plots for June-August 2007. Top row: Calibrated one-minute STEREO A (red) and B (blue) PLASTIC solar wind bulk velocity data. Second row: The same data as in the first row, but shifted by the radial and longitudinal time lag. Third row: Correlation between the A and time-shifted B bulk velocity data over one-third of a Carrington rotation time period $(\mathrm{CR} / 3)$. Fourth row: Regression plot of the $\mathrm{A}$ and time-shifted B bulk velocity data. 
spacecraft trajectories (Figures 1 and 2). For a radial separation $\Delta s$, with the measured solar wind bulk velocity by the $\mathrm{B}$ spacecraft, $u_{\mathrm{B}}$, the difference in the arrival times between the $\mathrm{A}$ and $\mathrm{B}$ spacecraft trajectories was calculated as

$$
\Delta t=\Delta s / u_{\mathrm{B}}
$$

which is shown in Figure 2 (bottom panel). For each one-minute measurement interval the time of measurements on the B spacecraft is shifted by $\Delta t$ as if they were taken at the radial distance of the spacecraft A:

$$
t_{\mathrm{Bs}}=t_{\mathrm{B}}-\Delta t .
$$

The theoretical time lag between a solar wind measurement by $\mathrm{A}$ at time $t_{\mathrm{A}}$ and the radially time-shifted measurement of solar wind from the same solar source by B at time $t_{\mathrm{Bs}}$ was derived from the longitudinal spacecraft separation, $\alpha$, and the solar rotation rate, $\omega$. This longitudinal time lag is given by

$$
t_{r}=\alpha / \omega,
$$

which is shown in Figure 2 (bottom panel). The total time shift, $t_{r}-\Delta t$, can be either positive or negative as the effect by longitudinal separation of the spacecraft is comparable to the influence of the spacecraft radial position difference. The bottom panel in Figure 2 shows the two time lags, $t_{r}$ and $\Delta t$, for the whole investigated time period. The $t_{r}$ rotational time lag (black) increases as the longitudinal spacecraft separation increases with time, whereas the $\Delta t$ radial time lag (violet) varies with the solar wind velocity and the radial spacecraft separation. Early in the mission, the radial separation between the two spacecraft causes a greater time lag than their longitudinal separation, therefore the solar wind first arrives at the A spacecraft, which is nearer the Sun, and somewhat later at the B spacecraft. Later, as the longitudinal separation increases, the features of the same solar wind origin are recorded first by the B spacecraft, and just somewhat later by the A spacecraft as the Sun rotates in that direction.

The STEREO A and B datasets of solar wind plasma were harmonized for the spacecraft radial and longitudinal separations by adjusting for this theoretical time lag. We searched available A spacecraft plasma measurements for the expected arrival time of a plasma parcel detected at spacecraft $\mathrm{B}$ at $t_{\mathrm{B}}$ within $\pm 30 \mathrm{~s}$ tolerance (tol):

$$
t_{\mathrm{A}}=\left(t_{\mathrm{B}}-\Delta t+t_{r}\right) \pm \text { tol. }
$$

From this procedure we obtained A and B solar wind bulk velocity data pairs theoretically corresponding to the same solar origin (Figure 3, second row). The observations are at oneminute cadence for both probes and we apply the strong constraint of $\pm 30 \mathrm{~s}$ tolerance at our A and time-shifted B data pair selection, though the data loss remains low thanks to the small number of data gaps. As a result, there is no need for interpolation; we have sufficient data remaining. Note also that the applied bulk velocity dataset contains the original values; no smoothing or despiking was needed because of the good quality of our measurements.

If the solar wind source was constant, the two datasets would be identical. Comparison of the original (Figure 3, first row) and the time-lag-corrected measurements (Figure 3, second row) shows clearly a better agreement between the A and B datasets after the timelag adjustment. This time-lag method allows us to investigate the time scales over which the solar wind bulk velocity evolves. This is done in the following section. 


\section{Results and Discussion}

We performed a correlation and regression analysis between the A and time-shifted B datasets. Correlation of this time-lag-corrected STEREO/PLASTIC solar wind bulk velocity data was performed by using the numerical correlation function

$$
r_{a b}=\frac{n \cdot \sum_{i} a_{i} \cdot b_{i}-\sum_{i} a_{i} \cdot \sum_{i} b_{i}}{\sqrt{n \cdot \sum_{i} a_{i}^{2}-\left(\sum_{i} a_{i}\right)^{2}} \cdot \sqrt{n \cdot \sum_{i} b_{i}^{2}-\left(\sum_{i} b_{i}\right)^{2}}},
$$

where $r_{a b}$ is the correlation coefficient, $n$ is the number of observations during the time period over which the correlation is calculated, $i$ is the running index of the summation, and $a_{i}$ and $b_{i}$ are the time-lag-corrected solar wind bulk velocity measurements by the A and B spacecraft, respectively. The observations are spaced one minute apart and the correlation analyses are performed for different time periods. Figure 3 (third row) shows the correlation between the two time-lag-corrected datasets using a time window of one-third of a Carrington rotation $(\mathrm{CR} / 3)$.

In general, for all time windows the correlation is large but decreasing with the longitudinal spacecraft separation, which means that there is a longer time for the temporal evolution of the solar wind between the two measurements. Although this decreasing tendency is clearly visible, the correlation coefficient is still around 0.85 for a 2-day time lag: the solar wind is rather persistent.

Regression plots between the time-lag-corrected datasets are shown in the bottom row of Figure 3. We find a good match between the velocities measured at the two spacecraft for the six months of observations. The individual points in the regression plot are around the line with slope 1. (Note that only contours are shown in the plot owing to the large amount of data.) This nice organization of the data becomes less clear for later time periods, but it is still visible even in the plot for August 2007, where the longitudinal spacecraft separation is about $25^{\circ}$. In summary, the regression analysis supports the results from the correlation analysis that the solar wind persistence is rather high at low solar activity.

There are some exceptions to this good correlation, and these are found during transient events in the solar wind. For example, there is a significant difference in the A and B solar wind data at the end of May 2007 ( DOY 142) as consequences of coronal mass ejection (CME) events. Clearly, CMEs do not qualify as a constant solar wind source on the solar wind surface, which is reflected in a lower correlation coefficient for such a plasma population. As another example, in the beginning of June ( $\sim$ DOY 155$)$ the correlation decreased most probably in connection with a corotating interaction region (CIR) event. On 20 July 2007 (DOY 201) the velocity increase measured by B is greater than by A, which is due to an exceptionally high latitudinal velocity gradient along the $2.2^{\circ}$ latitudinal separation of the two spacecraft (Rouillard et al., 2009). For the time period around DOY $231 \pm 4$ we have found very poor correlation between the A and B velocities. In the time period of DOY $227-232$ the disagreement is due to temporal evolution of the solar wind since the structure is evolving from B to A and then the next Carrington rotation further. The mismatch during the time period of DOY 232-235 is due to spatial effect since the same structure is conserved even in the next Carrington rotation.

To study different scales in the solar wind we investigate different time windows in the correlation analysis. A time window of one Carrington rotation (CR) delivers information about the temporal evolution of the whole Sun. CR/2 treats solar wind emerged just from half of the Sun. CR/3 (the same data as in Figure 3, third row) was chosen because there were about three clear fast solar wind features per Carrington rotation in the investigated 

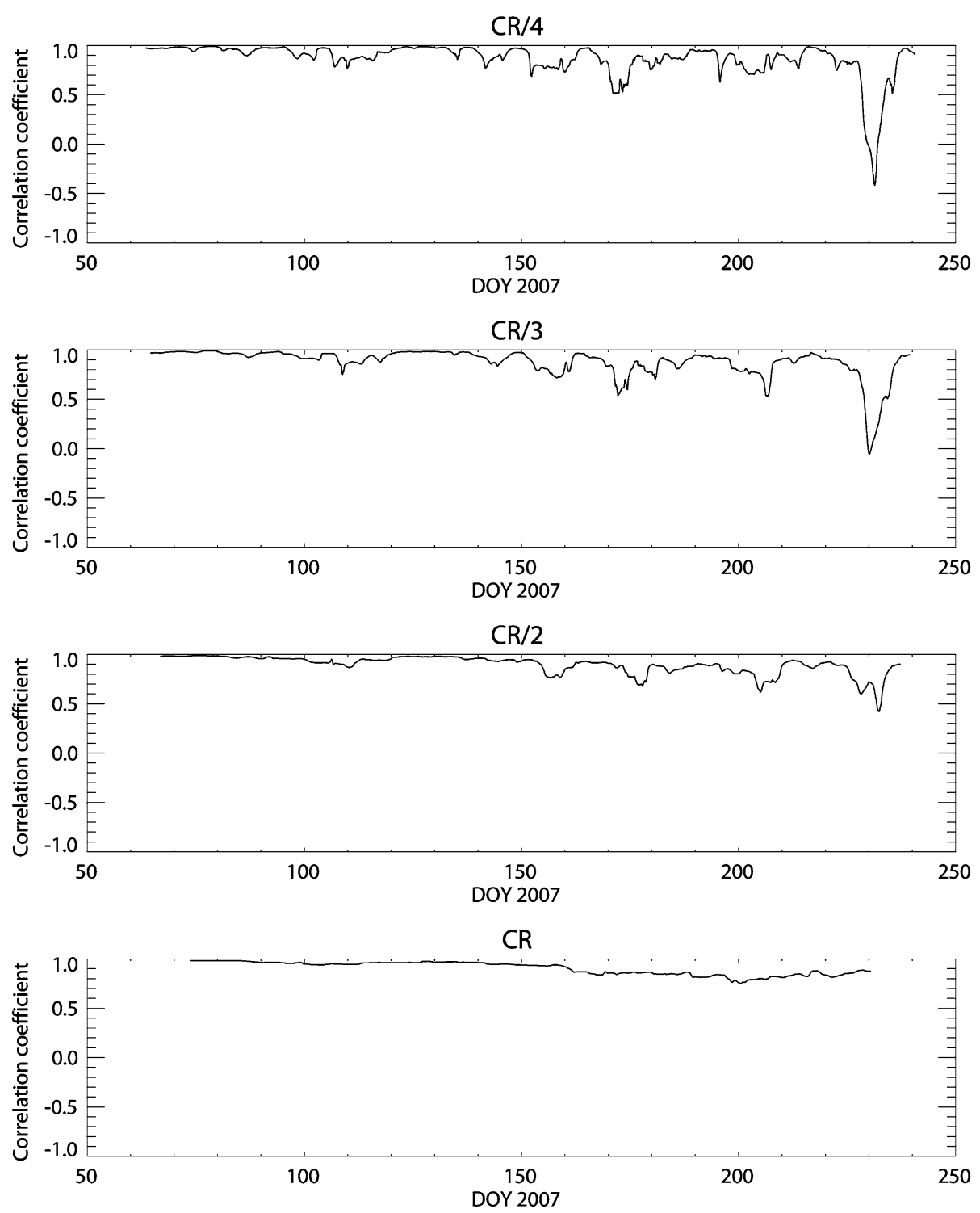

Figure 4 Correlation over different time windows between STEREO A and time-shifted B PLASTIC solar wind bulk velocity data for March-August 2007 with one-minute resolution. Top panel: One-quarter Carrington rotation $(\mathrm{CR} / 4)$. Second panel: One-third Carrington rotation $(\mathrm{CR} / 3)$. Third panel: One-half Carrington rotation $(\mathrm{CR} / 2)$. Fourth panel: One Carrington rotation $(\mathrm{CR})$. The correlation coefficient is always plotted in the center of the applied time period; for instance, the correlation coefficient on DOY 100 in the top panel $(\mathrm{CR} / 4)$ was calculated from measurements between DOY $100-\mathrm{CR} / 4 / 2$ and DOY 100 + CR/4/2.

dataset. CR/4 is about one week and allows the study of smaller scale solar wind features. The correlation results calculated with the four different time intervals are shown in Figure 4 for the whole investigated time period. For increasing length of this time window, which corresponds to larger spatial scales, the correlation data become smoother since the temporal 

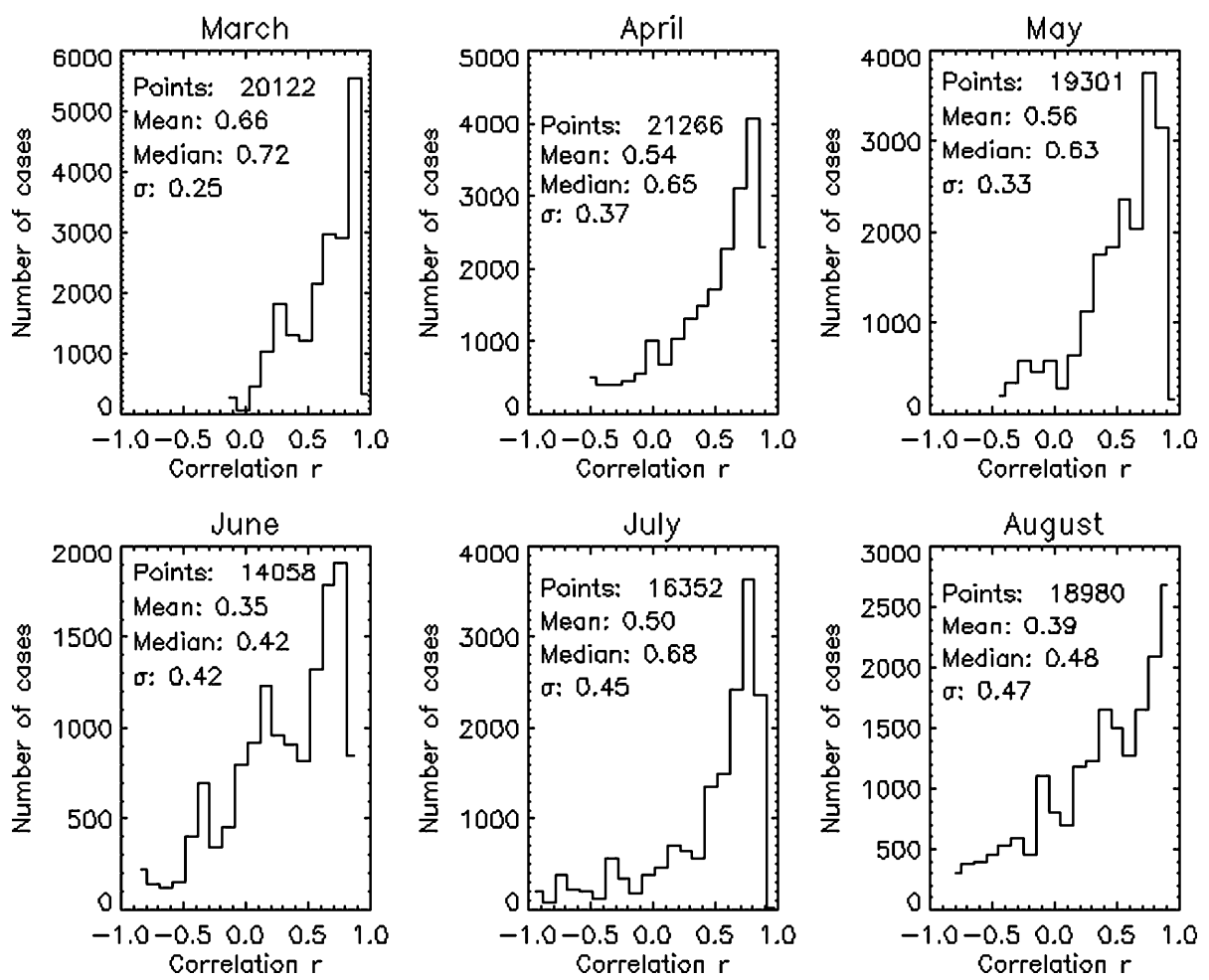

Figure 5 Histograms of the correlation coefficients of STEREO/PLASTIC solar wind bulk velocity observations. One-minute time resolution data over a time interval of one day were used.

evolution of smaller scales is averaged. For a time window of a full Carrington rotation, even the large difference in the A and B datasets around DOY 231 is smoothed out. In order to have a probability of more than $95 \%$ that the data pairs are correlated, the absolute value of the correlation coefficient has to be higher than 0.25 for a time interval of a quarter CR, or higher than 0.2 for a time interval of one CR, respectively. So even for the shortest time interval only the time period DOY 227 - 232 showed evidence for being uncorrelated.

Coplan et al. (2001) used time windows of 2 and 6 hours in their analysis. They use 96-s time resolution 2-hour observation interval data and 300-s time resolution 6-hour observation interval data, the latter showing better correlation (see Figure 4, Figure 5 and Table 2 in Coplan et al., 2001), which is a result of smaller random data component and more solar wind plasma parameter changes during this longer time period. In Figure 5 we show similar plots obtained from the data presented in this paper. The time interval was always one day since smaller intervals did not show reasonable results because of poor statistics. It can be seen that the mean and the median of the correlation coefficient both decrease with time with the exception of those in July. This supports the observation presented in Figure 3.

The observed correlation is high, and as expected it decreases with increasing time lag (Figure 6). However, the correlation coefficient is still about 0.85 for a time lag of 2 days. Using also STEREO/PLASTIC measurements Podesta, Galvin, and Farrugia (2008) found much lower correlations between the two bulk velocity datasets, mainly because they did not account for the temporal and spatial effects by applying the time-lag method described in the previous section. Gosling and Bame (1972) found a correlation of about 0.64 for 1-day 
Figure 6 STEREO A and B

PLASTIC solar wind bulk velocity correlation results calculated over a time window of one Carrington rotation as a function of the time lag from the longitudinal separation of the two STEREO spacecraft.

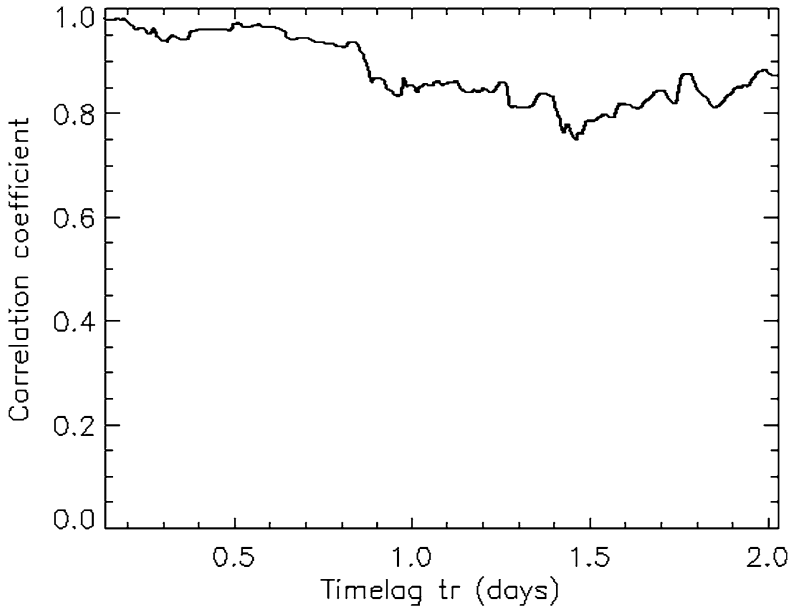

time lag by autocorrelation analysis, whereas we have found a value higher than 0.8 by the two-spacecraft correlation analysis.

\section{Conclusions}

We have analyzed solar wind bulk velocity measurements by the two STEREO spacecraft for the 6-month time period between March and August 2007. This period corresponds to quiet solar wind conditions, which is favorable to study the temporal evolution of the solar wind flow. The two solar wind bulk velocity datasets of one-minute time resolution measured by the two PLASTIC plasma instruments onboard STEREO A and B are well comparable after adjusting for the time lag from the different spacecraft positions. Since the longitudinal spacecraft separation is monotonically increasing from $1.2^{\circ}$ to $28.2^{\circ}$, the time difference $\left(t_{r}\right)$ between STEREO A and B measurements of the solar wind from the same solar source is also increasing from 0.09 up to 2.14 days. As a result, the temporal evolution of the solar wind can be studied for this time range. Some studies of multispacecraft correlation of solar wind plasma parameters, including velocity, have already been done before, but none have utilized such widely spaced spacecraft as here.

First, we time-shifted the B spacecraft data by the theoretically calculated time lag to forecast (or pastcast in case of later arrival at B) the A spacecraft measurements. Then we compared the forecasted and measured values by correlation and regression analysis and found a very good agreement for these spatial and temporal scales. As a result, we conclude that the solar wind can be considered as persistent for a period of at least 2 days during solar minimum. The correlation decreases as a function of the time lag (Figure 6); the coefficient is $\sim 0.85$ for a 2 -day time lag.

The correlation coefficients decreased as the time window decreased, which suggests that the temporal evolution depends on the spatial scales. Study of lower spatial scales results in lower correlations which is due to faster temporal evolution. We have also shown that as long as quiet conditions provide good agreement, transient events cause a visible difference between measurements at two different positions and time points.

The investigation of the solar wind persistence is important to estimate the reliability of the STEREO/PLASTIC solar wind bulk velocity forecast for Earth in the framework of 
space weather forecasting. This study will be extended in the future for separation distances of $0^{\circ}$ up to $180^{\circ}$ that will be reached by the two STEREO spacecraft after four years of operation. This will provide a full scan of the time lag.

Acknowledgements The authors would like to thank Jack T. Gosling and Alexis P. Rouillard for helpful discussions, as well as Christopher T. Russell for his comments. This work was supported by the Swiss National Fund for the University of Bern. It was also supported by the NASA STEREO/PLASTIC Grant for the University of New Hampshire and by a CNES Grant for CESR (CNRS-UPS). The authors acknowledge contributions from the entire STEREO group.

\section{References}

Coplan, M.A., Ipavich, F., King, J., Ogilvie, K.W., Roberts, D.A., Lazarus, A.J.: 2001, J. Geophys. Res. 106, 18615.

Galvin, A.B., Kistler, L.M., Popecki, M., Farrugia, C.J., Simunac, K.D.C., Ellis, L., et al.: 2008, Space Sci. Rev. 136, 437.

Gosling, J.T., Bame, S.J.: 1972, J. Geophys. Res. 77, 12.

Gosling, J.T., Asbridge, J.R., Bame, S.J., Feldman, W.C.: 1976, J. Geophys. Res. 81, 5061.

Kaiser, M.L., Kucera, T.A., Davila, J.M., St. Cyr, O.C.: 2007, Space Sci. Rev. 136, 5.

Karrer, R.: 2007, PhD thesis, University of Bern.

King, J.H., Papitashvili, N.E.: 2005, J. Geophys. Res. 110, A02104.

Opitz, A.: 2007, PhD thesis, University of Bern.

Paularena, K.I., Zastenker, G.N., Lazarus, A.J., Dalin, P.A.: 1998, J. Geophys. Res. 103, 14601.

Podesta, J.J., Galvin, A.B., Farrugia, C.J.: 2008, J. Geophys. Res. 113, A09104.

Richardson, J.D., Dasevisky, F., Paularena, K.I.: 1998, J. Geophys. Res. 103, 14619.

Rouillard, A.P., Savani, N., Davies, J.A., Lavraud, B., Forsyth, R.J., Morley, S.K., et al.: 2009, Solar Phys., submitted. 